\title{
Article
}

\section{Light Trapping in Single Elliptical Silicon Nanowires}

\author{
Wenfu Liu ${ }^{1,2, * \mathbb{D}}$, Yinling Wang ${ }^{1}$, Xiaolei Guo ${ }^{1}$, Jun Song ${ }^{1}$, Xiao Wang ${ }^{2}$ and Yasha $\mathrm{Yi}^{2,3, *}$ \\ 1 School of Mechanical and Energy Engineering, Huanghuai University, Zhumadian, Henan 463000, China; \\ wangyinling@huanghuai.edu.cn (Y.W.); guoxiaolei@huanghuai.edu.cn (X.G.); \\ songjun@huanghuai.edu.cn (J.S.) \\ 2 Integrated Nano Optoelectronics Laboratory, University of Michigan, Dearborn, MI 48128, USA; \\ xwa@umich.edu \\ 3 Energy Institute, University of Michigan, Ann Arbor, MI 48109, USA \\ * Correspondence: liuwenfu@huanghuai.edu.cn (W.L.); yashayi@umich.edu (Y.Y.)
}

Received: 28 September 2020; Accepted: 23 October 2020; Published: 25 October 2020

\begin{abstract}
Light trapping in single nanowires (NWs) is of vital importance for photovoltaic applications. However, circular NWs (CNWs) can limit their light-trapping ability due to high geometrical symmetry. In this work, we present a detailed study of light trapping in single silicon NWs with an elliptical cross-section (ENWs). We demonstrate that the ENWs exhibit significantly enhanced light trapping compared with the CNWs, which can be ascribed to the symmetry-broken structure that can orthogonalize the direction of light illumination and the leaky mode resonances (LMRs). That is, the elliptical cross-section can simultaneously increase the light path length by increasing the vertical axis and reshape the LMR modes by decreasing the horizontal axis. We found that the light absorption can be engineered via tuning the horizontal and vertical axes, the photocurrent is significantly enhanced by $374.0 \%(150.3 \%, 74.1 \%)$ or $146.1 \%(61.0 \%, 35.3 \%)$ in comparison with that of the CNWs with the same diameter as the horizontal axis of $100(200,400) \mathrm{nm}$ or the vertical axis of $1000 \mathrm{~nm}$, respectively. This work advances our understanding of how to improve light trapping based on the symmetry breaking from the CNWs to ENWs and provides a rational way for designing high-efficiency single NW photovoltaic devices.
\end{abstract}

Keywords: silicon; single nanowires; elliptical cross-section; absorption; photocurrent

\section{Introduction}

Single nanowire (NW) solar cells have increasingly attracted attention in recent years due to the following reasons [1-8]. Firstly, single NW solar cells can serve as nanoscale power sources integrated seamlessly with nanoelectronics. Secondly, results from single NWs can subsequently provide feedback for the design of new functional NWs. Finally, single NWs can assemble to form NW arrays, and results from single NWs can help understand the self-assembled NW-based solar cells [7-16].

Light trapping in single NW solar cells is very important for ensuring both high absorption and little photoactive material, which can enhance the absorption by increasing the light path length within the cell while it is possible to use less material in the cell [17-27]. Therefore, light trapping is an effective way to improve light absorption and enhance the photoelectric conversion efficiency of single NW solar cell. It is well known that the strong interaction between the incident light and a single NW has been applied to dramatically increase light trapping due to the leaky mode resonances (LMRs) [28-30]. However, the overall light-trapping performance of a single NW is still far below expectations owing to the narrow resonant peaks.

Therefore, a great variety of strategies have been employed to improve light-trapping ability. It has been shown that the light-trapping performance could be readily engineered by controlling the size, geometry and orientation of the NWs [31-37]. Our previous studies [25,38,39] showed that 
the light-trapping performance could be further improved by introducing a non-absorbing dielectric shell (or graded dual shells) as the antireflection coating, which was experimentally and numerically demonstrated in the recent studies [40-44]. Recently, some new strategies have been implemented to improve the light-trapping capability of the NWs based on the symmetry breaking. For example, front (or rear)-opening crescent design [45,46], off-axial core-shell design [47,48], asymmetrical nanovoid design [33], partially capped design [49-52], nanocone design [53-56], inclined design [57-59] and disorder design [60-64]. Comparing with the circular NWs (CNWs), elliptical NWs (ENWs) provide more possibility to tune light trapping. It is worth noting that the elliptical nanostructures have been applied to improve light trapping in single ellipsoids [65,66], NW arrays [67], nanocone arrays [68] and nanohole arrays [61,69]. However, to the best of our knowledge, very few investigations based on the symmetry breaking from the CNW to ENW have been explored to improve light trapping in single NWs so far.

In this work, we carry out detailed investigations on the light-trapping effect of single ENWs. We demonstrate that the giant enhancement of the light absorption occurs when the ENWs replace the CNWs. The detailed analysis of the electric field, absorption mode profile and photogeneration rate shows that this enhancement is mainly attributed to the asymmetry breaking from the CNWs to ENWs. Specifically, the light path length can be increased by increasing the vertical axis and the LMR modes can be reshaped by decreasing the horizontal axis. Simulation results reveal that the photocurrent is significantly enhanced by $374.0 \%(150.3 \%, 74.1 \%)$ or $146.1 \%(61.0 \%, 35.3 \%)$ compared with that of the CNW with the same diameter as the horizontal axis of $100(200,400) \mathrm{nm}$ or the vertical axis of $1000 \mathrm{~nm}$, respectively.

\section{Model and Methods}

\subsection{Model}

Figure 1 schematically illustrates the cross-sectional views of a CNW and several ENWs. The horizontal $(x)$ and vertical $(y)$ axes of the ENWs are denoted by $a$ and $b$. The horizontal (or vertical) axis is perpendicular (or parallel) to the light illumination direction, as shown using the colorful arrows in Figure 1. The values of $a$ are chosen to be 400, 200 and $100 \mathrm{~nm}$ as the typical representative nanoscale size and the values of $b$ are chosen to the range from 10 (oblate ENWs) to 1000 (prolate ENWs) nm. It should be noted that the CNWs are also shown for comparison, where the diameter $D$ of the CNWs is chosen to be $b$ to investigate the improved light trapping due to the reshaped LMRs by decreasing $a$, or $a$ to investigate the improved light trapping owing to the increased light path length by increasing $b$. Silicon is chosen as a typical semiconductor material and its wavelength-dependent refractive index is adopted from the experimental data [70].

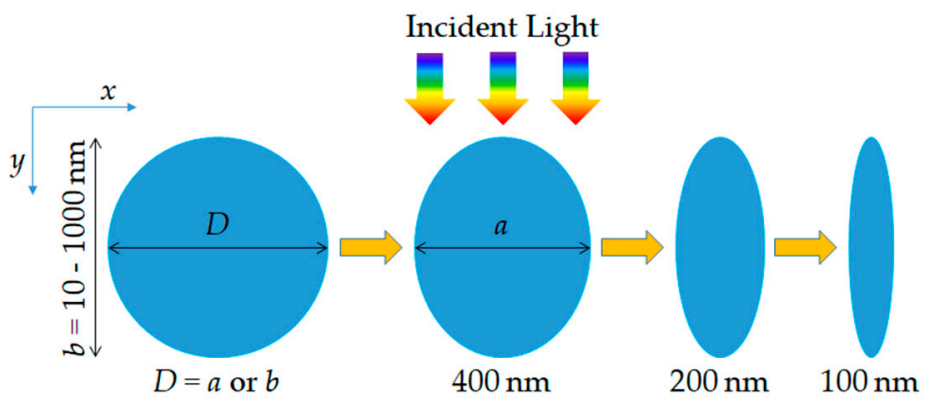

Figure 1. Schematics of the cross-section of a circle nanowire (CNW) and several elliptical nanowires (ENWs). The geometrical metrics used to describe the geometry of the ENWs, the horizontal ( $x$ ) axis $a$ and the vertical ( $y$ ) axis $b$, are shown. Note here that three representative values of $a$ are chosen to be 400,200 and $100 \mathrm{~nm}$, the range of $b$ is from 10 to $1000 \mathrm{~nm}$. The diameter $D$ of the CNWs are chosen to be $a$ or $b$ for comparison, the material of the ENWs is set to be silicon as a representative semiconductor and the light illumination is perpendicular to the axis of the ENW from above. 


\subsection{Methods}

Numerical simulations are carried out by solving Maxwell's equations based on the two-dimensional finite difference time domain (2D FDTD) method [71-73] by assuming that the length of the NWs is infinitely long, i.e., its length is far larger than the size of the cross-section, which can be referred to the work of Kim and co-workers for details [35-37,42]. In this simulation, the ENWs are illuminated perpendicularly by sunlight from the top, the wavelength range of the incident light is from 300 to $1100 \mathrm{~nm}$ with a step size of $5 \mathrm{~nm}$ considering solar radiation and the bandgap of silicon, the perfectly matched layers (PML) boundary conditions are applied to avoid any non-physical reflection with the boundaries, the total-field scattered-field (TFSF) method was adopted to ensure that a single NW interacts with an infinite plane wave. Also, the minimum cell size of the FDTD mesh is set to from 0.05 to $5 \mathrm{~nm}$ corresponding to $b(5 \rightarrow 1000 \mathrm{~nm})$ to guarantee the accuracy of the simulation results.

\subsubsection{The Normalized Electric Field $\left(\mathrm{E}_{\mathrm{r}}\right)$}

The normalized electric field $\left(E_{r}\right)$ can be defined as [49]:

$$
E_{r}=E / E_{0}
$$

where $E$ is the electric field of the ENWs, which is obtained by FDTD numerical simulation, and $E_{0}$ is the electric field of the solar incident light, respectively.

\subsubsection{The Absorption Mode Profile ( $\left.\mathrm{P}_{\mathrm{abs}}\right)$}

The wavelength-dependent absorption mode profile $\left(P_{\text {abs }}\right)$ calculated from the Poynting theorem can be expressed as $[44,51,56,74]$ :

$$
P_{a b s}=\frac{1}{2} \omega \varepsilon^{\prime \prime}|E|^{2},
$$

where $\omega$ is the angular frequency of the incident light and $\varepsilon^{\prime \prime}$ is the imaginary part of the permittivity of silicon, respectively.

\subsubsection{The Absorption Efficiency $\left(\mathrm{Q}_{\mathrm{abs}}\right)$}

To qualify the light-trapping performance of the ENWs, we define the absorption efficiency $\left(Q_{\mathrm{abs}}\right)$ as $[44,51,56,74]$ :

$$
Q_{\mathrm{abs}}=C_{\mathrm{abs}} / C_{\text {geo }},
$$

where $C_{\text {geo }}$ is the projected area of the ENWs and $C_{\mathrm{abs}}$ is the absorption cross-section per unit length obtained by,

$$
C_{\mathrm{abs}}=\frac{\iint P_{a b s} d x d y}{I_{0}}=k_{0} \varepsilon^{\prime \prime}{ }_{r} \iint\left|E_{r}\right|^{2} d x d y,
$$

where $k_{0}$ is the wave vector in air, $\varepsilon^{\prime \prime}{ }_{r}$ is the imaginary part of the relative permittivity of silicon, $x$ and $y$ are the coordinate axes shown in Figure 1 , and $I_{0}$ is the solar incident light intensity expressed as $[44,51,56,74]$ :

$$
\begin{gathered}
I_{0}=\frac{1}{2} c \varepsilon_{0}\left|E_{0}\right|^{2}, \\
\varepsilon^{\prime \prime}{ }_{r}=\varepsilon^{\prime \prime} / \varepsilon_{0},
\end{gathered}
$$

where $c$ is the speed of light and $\varepsilon_{0}$ is the permittivity in air, respectively. 
2.2.4. The Photogeneration Rate $(G)$

The spatially dependent photogeneration rate $(G)$ is readily calculated by $[26,75]$ :

$$
G=\int_{300}^{1100} \frac{P_{a b s}}{\hbar \omega} d \lambda=\int_{300}^{1100} \frac{\varepsilon^{\prime \prime}|E|^{2}}{2 \hbar} \mathrm{d} \lambda,
$$

where $\hbar$ is the reduced Planck's constant and $\lambda$ is the wavelength of the incident light. Note that when using Equation (7), each photon absorbed in ENW contributes to the photocurrent without considering recombination losses.

\subsubsection{The Ultimate Photocurrent $\left(\mathrm{J}_{\mathrm{ph}}\right)$}

The overall light-trapping performance is evaluated using the ultimate photocurrent $\left(J_{\mathrm{ph}}\right)$ calculated by:

$$
J_{\mathrm{ph}}=\frac{q}{C_{g e o}} \iint G d x d y=q \int_{300}^{1100} \Gamma(\lambda) Q_{a b s}(\lambda) d \lambda,
$$

where $q$ is the elementary charge and $\Gamma$ is the AM1.5G standard solar photon flux density spectrum. It should be noted here that $100 \%$ collection efficiency is assumed, which has been widely employed to evaluate the ultimate photocurrent $[29,75]$.

\subsubsection{The Photocurrent Enhancement Factor (PEF)}

The photocurrent enhancement is evaluated by employing the photocurrent enhancement factor (PEF) using the relation:

$$
\mathrm{PEF}=\left(J_{\mathrm{ph}, \mathrm{ENWs}}-J_{\mathrm{ph}, \mathrm{CNWs}}\right) / J_{\mathrm{ph}, \mathrm{CNWs}},
$$

where $J_{\mathrm{ph}, \mathrm{ENWs}}$ and $J_{\mathrm{ph}, \mathrm{CNWs}}$ are the photocurrent density for the ENWs and CNWs, respectively.

\section{Results and Discussion}

\subsection{Light-Trapping Mechanism in Single Elliptical Nanowire (ENW)}

To understand the light-trapping mechanism responsible for the improved absorption of the ENW, we investigate the absorption efficiency $\left(Q_{\mathrm{abs}}\right)$, ultimate photocurrent $\left(J_{\mathrm{ph}}\right)$, normalized electric field $\left(E_{r}\right)$, absorption mode profile $\left(P_{\text {abs }}\right)$ and photogeneration rate $(G)$, respectively. Note here that $a=200$, $b=500 \mathrm{~nm}$ and $D=a=200$ or $D=b=500 \mathrm{~nm}$ denotes the cases of the CNW with the same $a$ or $b$ for comparison, respectively.

\subsubsection{The Absorption Efficiency $\left(\mathrm{Q}_{\mathrm{abs}}\right)$}

To quantitatively characterize the light-trapping performance of the ENW in comparison with the CNW, we first examine the absorption spectra obtained by Equation (3). In Figure 2, we show $\lambda$-dependent $Q_{\mathrm{abs}}$ spectra of the ENW with $a=200$ and $b=500 \mathrm{~nm}$ and the CNW with $D=b=500 \mathrm{~nm}$ under normally-incident transverse-magnetic (TM), transverse-electric (TE) and unpolarized light, respectively. It is important to emphasize that the unpolarized illumination, like sunlight, is calculated by averaging TM and TE light illumination. TM and TE lights are shown in the inset in the top center of Figures $2 a$ and $2 b$, respectively. It is clear that $Q_{a b s}$ of the ENW is much bigger than that of the CNW in the short-wavelength range of $\lambda<\lambda_{\mathrm{cTM}} \sim 675, \lambda<\lambda_{\mathrm{cTE}} \sim 545$ or $\lambda<\lambda_{\mathrm{c}} \sim 670 \mathrm{~nm}$ for TM, TE or unpolarized light (except for several narrow peaks, for example, $\lambda=555 \mathrm{~nm}$ for unpolarized light), which can result in a significant photocurrent enhancement since the $Q_{\text {abs }}$ spectra of the CNW well matches the solar spectrum according to Equation (8). In comparison, the light absorption of the ENW seems to be comparable in the long-wavelength range of $\lambda>\lambda_{\mathrm{cTM}}, \lambda>\lambda_{\mathrm{cTE}}$ or $\lambda>\lambda_{\mathrm{c}}$, which can lead to a little contribution to the photocurrent enhancement. Note that $\lambda_{\mathrm{cTM}}, \lambda_{\mathrm{cTE}}$ and $\lambda_{\mathrm{c}}$ are the 
characteristic wavelengths, below which the light absorption is always enhanced and can be readily determined for a fixed $a, b$ and $D$.

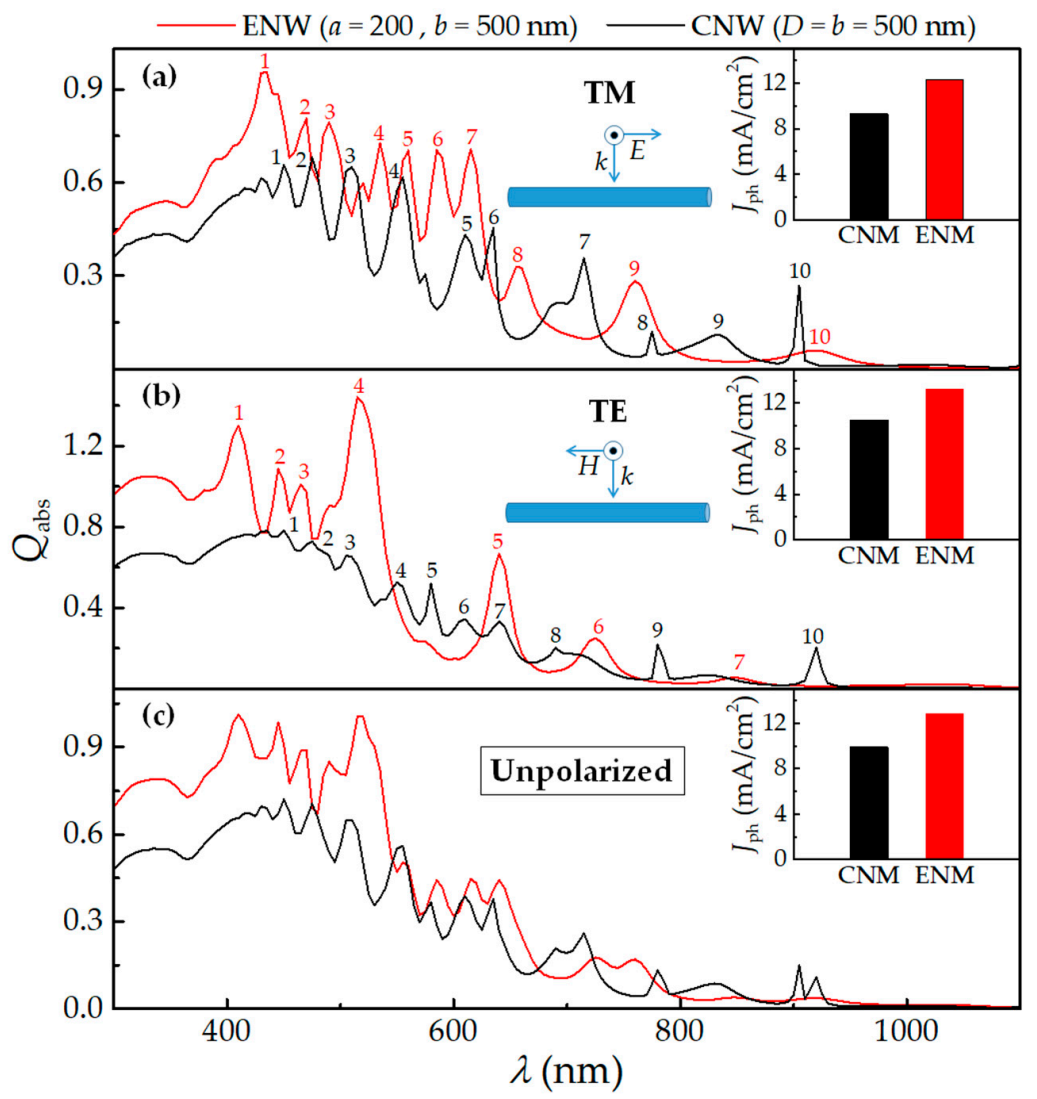

Figure 2. Absorption efficiency $\left(Q_{\mathrm{abs}}\right)$ of the CNW and ENW as a function of wavelength $(\lambda)$ for (a) transverse-magnetic (TM), (b) transverse-electric (TE) and (c) unpolarized light illumination, respectively. The insets at the top center illustrate the illumination geometries for (a) TM (electric field parallel to the axis of the ENW) and (b) TE (electric field perpendicular to the axis of the ENW) light illumination, respectively. Also, the insets at the top right corner show the ultimate photocurrent $\left(J_{\mathrm{ph}}\right)$ of the CNW and ENW for (a) TM, (b) TE and (c) unpolarized light, respectively. Note that $a=200$ and $D=b=500 \mathrm{~nm}$.

Moreover, the $Q_{\mathrm{abs}}$ spectra for TM light present some characteristics different from that for TE light. Specifically, compared to the $Q_{\text {abs }}$ spectra of the CNW with $D=b=500 \mathrm{~nm}$, the number of resonant peaks is increased $(5 \rightarrow 7)$ in the wavelength range of $\lambda<\lambda_{\mathrm{cTM}}$ due to the blue-shift of peaks and the light absorption is slightly improved for TM light, for example, $0.62 \rightarrow 0.73$ for the fourth peak, however, the number of resonant peaks is decreased $(10 \rightarrow 7)$ in the whole wavelength range and the light absorption is dramatically enhanced in the wavelength range of $\lambda<\lambda_{\mathrm{cTE}}$ at the same time, for instance, $0.53 \rightarrow 1.44$ for the fourth peak. Note here that some $Q_{\text {abs }}$ values for TE light exceed unity, which is attributed to the fact that the absorption cross-section is greater than the physical cross-section. All in all, the absorption results indicate the great potential of the single ENW in improving light trapping due to the symmetry-broken structure from the CNW to ENW.

\subsubsection{The Ultimate Photocurrent $\left(\mathrm{J}_{\mathrm{ph}}\right)$}

To evaluate the light-trapping performance of the ENW for photovoltaic applications, we then calculated the ultimate photocurrent $\left(J_{\mathrm{ph}}\right)$ according to Equation (8). For a direct comparison, in the insets of the upper right corner of Figure 1, we show $J_{\mathrm{ph}}$ of the ENW and CNW corresponding to $Q_{\mathrm{abs}}$ for TM, TE and unpolarized light illumination, respectively. It is observed that $J_{\mathrm{ph}}$ of the ENW is much 
bigger than that of the CNW with $D=b=500 \mathrm{~nm}$. $J_{\mathrm{ph}}$ for TM, TE and unpolarized light illumination reaches $12.32,13.24$ and $12.78 \mathrm{~mA} / \mathrm{cm}^{2}$, which is $32.5 \%, 26.6 \%$ and $29.4 \%$ higher than that of the CNW $\left(9.30,10.46\right.$ and $\left.9.88 \mathrm{~mA} / \mathrm{cm}^{2}\right)$, respectively. It is worth noting that the photocurrent enhancement is mainly ascribed to the reshaped LMRs caused by decreasing $a$ (that is $D \rightarrow a$, here $500 \rightarrow 200 \mathrm{~nm}$ ) compared to the CNW with $D=b=500 \mathrm{~nm}$, as discussed later. Moreover, $J_{\mathrm{ph}}$ can be significantly enhanced due to the increased light path length by increasing $b(D \rightarrow b$, here $200 \rightarrow 500 \mathrm{~nm})$ compared with the CNW with $D=a=200 \mathrm{~nm}$. $J_{\mathrm{ph}}$ is $50.2 \%, 82.9 \%$ and $65.5 \%$ higher than that of the CNW $(8.20,7.24$ and $7.72 \mathrm{~mA} / \mathrm{cm}^{2}$ ) [39] for TM, TE and unpolarized light illumination, respectively. The photocurrent results further indicate the huge potential of light trapping in single ENW for photovoltaic applications.

\subsubsection{The Normalized Electric Field $\left(\mathrm{E}_{\mathrm{r}}\right)$}

To understand the mechanism of light trapping in single ENW, we first examine the normalized electric field $\left(E_{r}\right)$ calculated by Equation (1). In Figure 3, we present the $E_{r}$ profiles of the CNW $(D=b$ $=500 \mathrm{~nm})$ and the ENW $(a=200$ and $b=500 \mathrm{~nm})$ corresponding to the positions denoted by Arabic numerals in Figure 2a,b under TM and TE light illumination, respectively. Note that Figure 3a,c show the $E_{r}$ profiles of the CNW for TM $(\lambda=450,475,510,550,610,635,715,775,835$ and $905 \mathrm{~nm})$ and TE $(\lambda=450,475,505,550,580,610,640,690,780$ and $920 \mathrm{~nm})$ light illumination, while Figure $3 \mathrm{~b}$,d show those of the ENW for TM $(\lambda=435,470,490,535,560,585,615,655,760$ and $920 \mathrm{~nm})$ and TE $(\lambda=410$, $440,465,515,640,725$ and $850 \mathrm{~nm}$ ) light illumination, respectively.

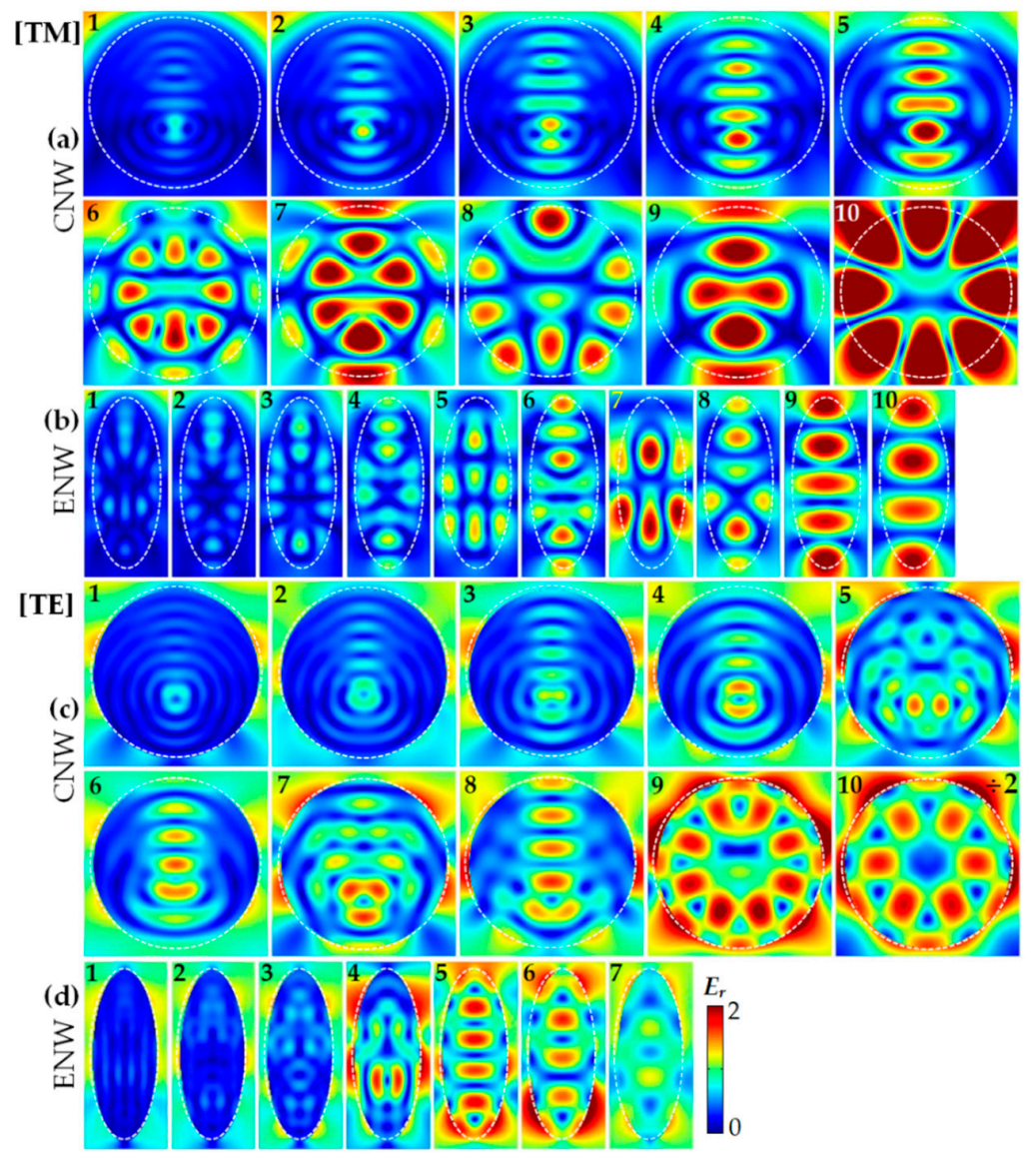

Figure 3. The representative normalized electric field $\left(E_{r}\right)$ profiles of the CNW and ENW at the absorption peaks indicated by Arabic numerals in Figure $2 a, b:(\mathbf{a}, \mathbf{b})$ for TM and (c,d) for TE light illumination (with an identical color scale); (a,c) for the CNW with $D=b=500 \mathrm{~nm}$ and $(\mathbf{b}, \mathbf{d})$ for the ENW with $a=200$ and $b=500 \mathrm{~nm}$, respectively. Note that the $E_{r}$ intensity in $\mathrm{c}(10)$ is properly shrunk for better illustration. 
It is observed that there are common characteristics of the $E_{r}$ profiles between the ENW and CNW. Firstly, the enhanced light trapping of the ENW is attributed to the excitation of the LMRs, likewise in CNW [28,29], which can confine light by multiple total internal reflections at the ENW/air interface when the wavelength of the incident light matches one of the LMRs supported by the ENW. The LMRs can be termed as $\mathrm{TM}_{m l}$ or $\mathrm{TE}_{m l}$, where $m$ and $l$ describe the azimuthal mode number and the radial order of the resonances, respectively. For example, the $E_{r}$ profiles of the ENW in Figure $3 b(10)$ and Figure $3 \mathrm{~d}(7)$ show more characteristics of the $\mathrm{TM}_{12}$ and $\mathrm{TE}_{11}$ modes of the CNW, respectively. Secondly, both NW configurations show much higher $E_{r}$ intensities in the long- than in the short-wavelength range. Specifically, the $E_{r}$ intensities for both NW configurations are much higher in Figure $3 a, b(5-10)$ than Figure $3 a, b(1-4)$ for TM light, while the $E_{r}$ intensities of the CNW in Figure $3 c(5-10)$ and the ENW in Figure $3 d(4-7)$ are much bigger than those of the CNW in Figure $3 c(1-4)$ and the ENW in Figure $3 d(1-3)$ for TE light, respectively, which is attributed to the fact that $\varepsilon^{\prime \prime}{ }_{r}$ is much smaller in the long- than short-wavelength range, resulting in a stronger resonance.

More importantly, there are many distinct characteristics of the $E_{r}$ profiles for both NW configures due to the symmetry breaking from the CNW to ENW. Firstly, the $E_{r}$ profiles of the CNW exhibit a large number of exact (for example, $\mathrm{TM}_{51}$ in Figure $3 \mathrm{a}(8)$ and $\mathrm{TE}_{13}$ in Figure $3 \mathrm{c}(9)$ ) and approximate degeneracies (for example, $\mathrm{TM}_{51}$ in Figure $3 \mathrm{a}(10)$ and $\mathrm{TE}_{13}$ in Figure $3 \mathrm{c}(10)$ ). In contrast, the $E_{r}$ profiles of the ENW exhibit different numbers, positions and modes of the resonant peaks between TM and TE light, indicating the higher tunability of light trapping in ENW than CNW. Secondly, the $E_{r}$ intensities at some resonant peaks are much larger only near the vertical axis of the CNW (for example, Figure 3a(1-5) for TM light and Figure $3 c(1-4,6,8)$ for TE light), while those at all resonant peaks are much bigger inside the whole ENW owing to the excitation of more complex LMRs in the short-wavelength range (for example, Figure 3b(1-4) for TM light and Figure 3d(1-3) for TE light), indicating the more vital interaction of incident light with the ENW, leading to a more significant light trapping comparison with the CNW.

\subsubsection{The Absorption Mode Profile $\left(\mathrm{P}_{\mathrm{abs}}\right)$}

To further understand the physics behind light trapping in single ENW, we then examine the absorption mode profile $\left(P_{\mathrm{abs}}\right)$ calculated by Equation (2). In Figure 4, we present the normalized $P_{\text {abs }}$ of the CNW $(D=b=500 \mathrm{~nm})$ and the ENW ( $a=200$ and $\left.b=500 \mathrm{~nm}\right)$ corresponding to the same positions denoted by Arabic numerals in Figures 2 and 3 under TM and TE light illumination, respectively. Note that Figure $4 \mathrm{a}, \mathrm{c}$ show the normalized $P_{\mathrm{abs}}$ of the $\mathrm{CNW}$ for TM and TE light, while Figure $4 \mathrm{~b}$,d show those of the ENW, respectively. It is observed that both NW configurations exhibit much higher absorption in the short- and medium- than long-wavelength range. Specifically, the light absorption for both NW configurations is much higher in Figure $4 a, b(1-7)$ than Figure $4 a, b(8-10)$ for TM light, while the light absorption of the CNW in Figure $4 c(1-7)$ and the ENW in Figure $4 d(1-5)$ is much bigger than that of the CNW in Figure $4 c(8-10)$ and the ENW in Figure $4 d(6-7)$ for TE light, respectively, which is attributed to the fact that $\varepsilon^{\prime \prime}{ }_{r}$ is much greater in the short- and medium- than long-wavelength range, leading to a stronger absorption in the short- and medium wavelength range.

It is worth noting that the match between $\varepsilon^{\prime \prime}{ }_{r}$ and $E_{r}$ becomes another essential factor in evaluating the absorption in the specific wavelength according to Equation (2). For instance, although the $E_{r}$ intensities of the ENW at $\lambda=\lambda_{10}=920 \mathrm{~nm}$ for TM light is much larger, the corresponding $\varepsilon^{\prime \prime}{ }_{r}$ is the smallest, which still leads to a lower absorption, while although those of the ENW at $\lambda=\lambda_{1}=410 \mathrm{~nm}$ for TM light is the smallest, the corresponding $\varepsilon^{\prime \prime}{ }_{r}$ is much larger, which results in a more significant absorption. It is readily observed that the spatially localized absorption sites of the ENW in both ends of the horizontal axis are considerably increased compared to the CNW, and more absorption sites appear and fill in the whole ENW. Such enhanced strong light-matter interaction results in giant light trapping, as shown in Figure 2. 


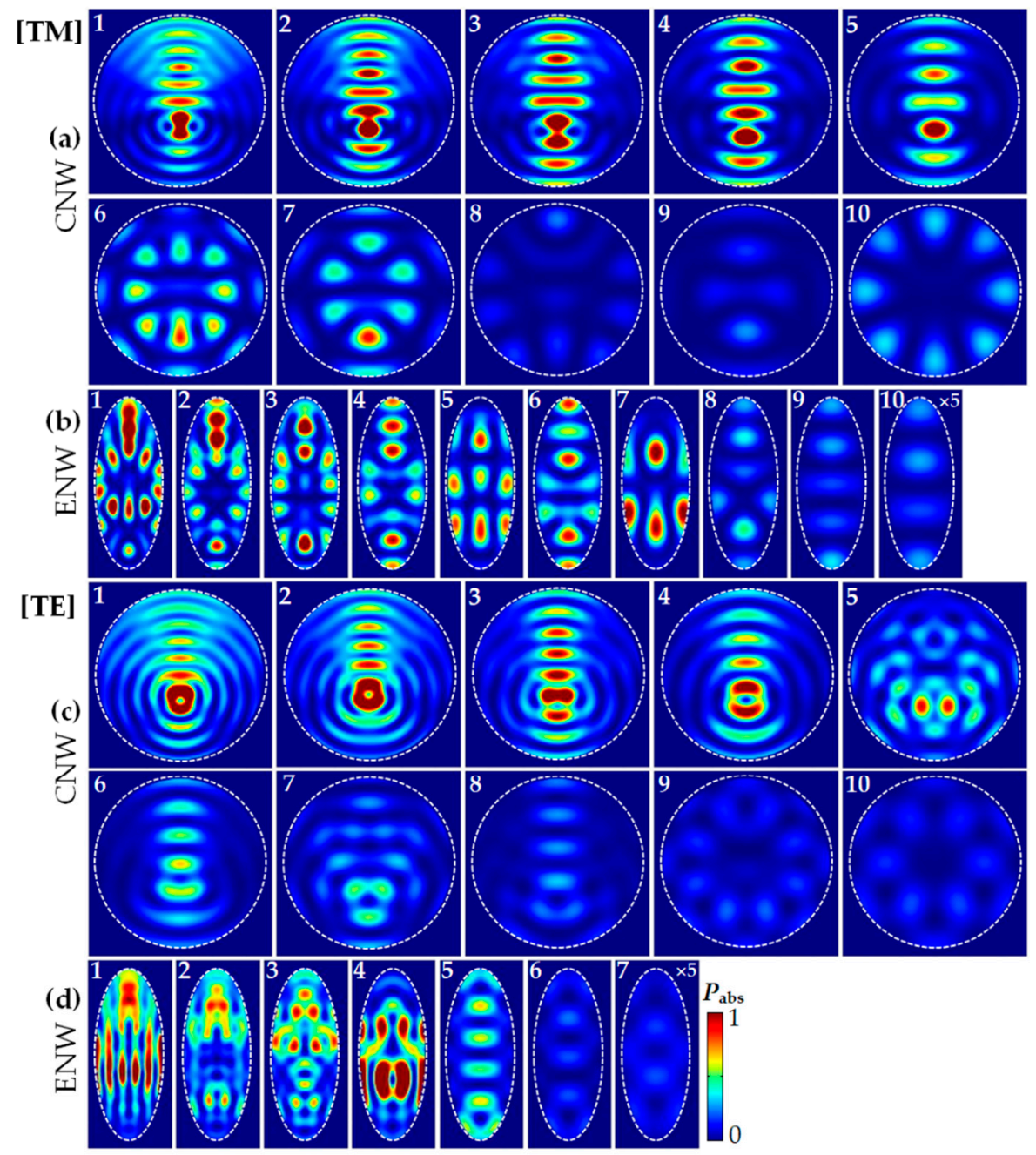

Figure 4. The representative normalized absorption mode profiles $\left(P_{\mathrm{abs}}\right)$ for the CNW and ENW at the absorption peaks indicated by Arabic numerals in Figure 2a,b and Figure 3: (a,b) for TM and (c,d) for TE light illumination (with an identical color scale); (a,c) for the CNW with $D=b=500 \mathrm{~nm}$ and $(\mathbf{b}, \mathbf{d})$ for the ENW with $a=200$ and $b=500 \mathrm{~nm}$, respectively. Note that $P_{\mathrm{abs}}$ in $\mathbf{b}(10)$ and $\mathbf{d}(7)$ are properly magnified for better illustration.

\subsubsection{The Photogeneration Rate $(\mathrm{G})$}

To further confirm the physical mechanism discussed above, we show the photogeneration rate $(G)$ calculated by Equation (6). In Figure 5, we present the normalized $G$ profiles of the CNW $(D=b=$ $500 \mathrm{~nm}$ ) and the ENW ( $a=200$ and $b=500 \mathrm{~nm}$ ) for TM and TE light illumination. Note that Figure $5 \mathrm{a}, \mathrm{c}$ show the normalized $G$ profiles of the CNW for TM and TE light, while Figure 5b,d show those of the ENW, respectively. It is observed in Figure $5 a, b$ that the absorption of the ENW is much stronger at both ends of the horizontal axis than that of the CNW for TM light. At the same time, it is observed in Figure 4c,d that the absorption of the ENW fills in the whole NW for TE light, consistent with the results discussed above. These results further demonstrate that this enhancement arises mainly from the excitation of more LMR modes caused by decreasing $a$ compared to the CNW with $D=b$. In other words, the ENW can better interact with the incident light compared to the CNW, leading to more resonant absorption sites appearing and filling in the whole ENW. 


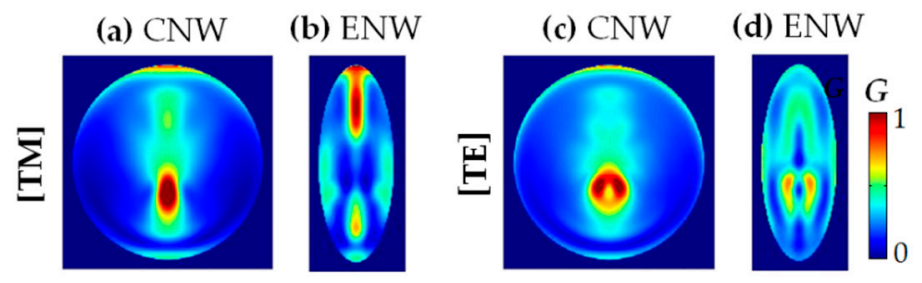

Figure 5. The normalized photogeneration rate $(G)$ profiles for the CNW and ENW: $(\mathbf{a}, \mathbf{b})$ for TM and $(\mathbf{c}, \mathbf{d})$ for TE light illumination (with an identical color scale); $(\mathbf{a}, \mathbf{c})$ for the $\mathrm{CNW}$ with $D=b=500 \mathrm{~nm}$ and $(\mathbf{b}, \mathbf{d})$ for the ENW with $a=200$ and $b=500 \mathrm{~nm}$, respectively.

\subsection{The Light-Trapping Performance of Single ENWs}

To verify that the improved light trapping is not just specific for the dimension discussed above, we calculate $Q_{\mathrm{abs}}$ and $J_{\mathrm{ph}}$ of the ENWs with $b=10 \rightarrow 1000 \mathrm{~nm}$ and $a=400,200$ and $100 \mathrm{~nm}$, and $Q_{\mathrm{abs}}$ and $J_{\mathrm{ph}}$ of the CNWs with $D=b$ are investigated for comparison.

\subsubsection{The Absorption Efficiency of Single ENWs}

To evaluate the light-trapping performance of the ENWs for photovoltaic applications, we first investigate the effect of its geometrical parameters on the absorption efficiency calculated using Equation (3). In Figure 6, we show 2D contour maps of $\lambda$-dependent $Q_{\text {abs }}$ as a function of $b(10 \rightarrow 1000 \mathrm{~nm})$ for the ENWs with $a=400,200$ and $100 \mathrm{~nm}$ for TM and TE light illumination, respectively. Note that the $Q_{\text {abs }}$ spectra of the CNWs with $D=b$ is also shown for comparison. It is observed that the $Q_{\text {abs }}$ spectra of the ENWs show the extensive wavelength tenability, and the light absorption of the NEWs with $b>$ $a$ is dramatically enhanced compared to that of the CNWs for both polarized lights.

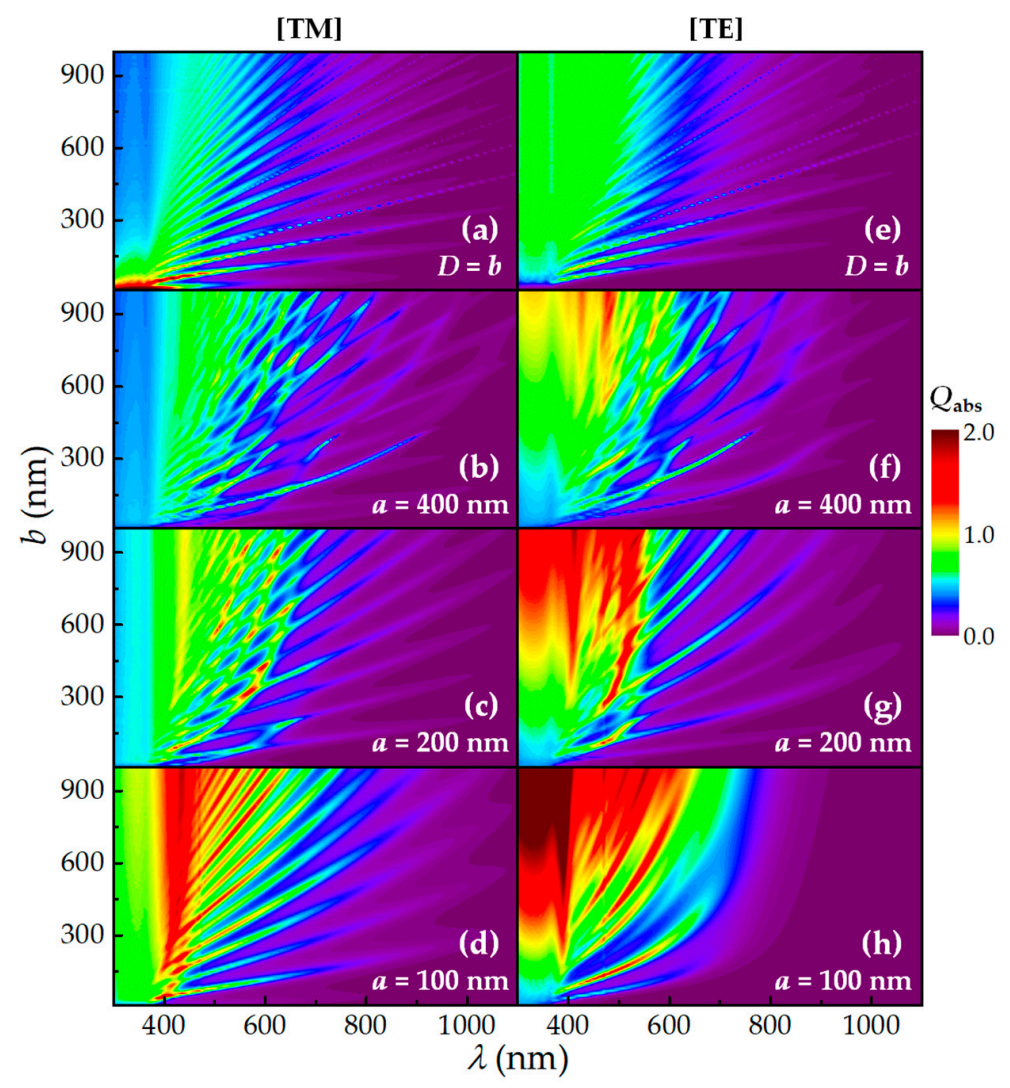

Figure 6. $Q_{\mathrm{abs}}$ versus $b$ and $\lambda$ for the CNWs and ENWs: (a-d) for TM and (e-h) for TE light illumination (with an identical color scale); (a,e) for the CNW with $D=b ;(\mathbf{b}, \mathbf{f}, \mathbf{c}, \mathbf{g}, \mathbf{d}, \mathbf{h})$ for the ENWs with $a=400$, 200 and $100 \mathrm{~nm}$, respectively. 
On the one hand, the $Q_{\mathrm{abs}}$ spectra of the ENWs exhibit some characteristics similar to the CNWs. Firstly, the number of resonant peaks increases. For example, Figure $6 \mathrm{~d}$ shows five and 11 peaks for the ENWs with $b=300$ and $900 \mathrm{~nm}$, respectively. Secondly, the peaks tend to red-shift with increasing $b$. For instance, in Figure $6 \mathrm{~d}$, the peak at near $\lambda=420 \mathrm{~nm}$ for the ENW with $b=300 \mathrm{~nm}$ can shift substantially to about $\lambda=800 \mathrm{~nm}$ for the ENW with $b=1000 \mathrm{~nm}$. Finally, the resonant peaks in different ENWs may originate from entirely different resonances. For example, Figure $6 \mathrm{~d}$ shows the peaks near $\lambda=600 \mathrm{~nm}$ for the ENWs with $b=300$ and $600 \mathrm{~nm}$.

On the other hand, the $Q_{\text {abs }}$ spectra of the ENWs also show some characteristics different from the CNWs. Firstly, the light absorption of the CNWs is gradually weakened due to the weak LMRs with increasing $D$. In contrast, the light absorption of the ENWs is significantly enhanced with increasing $b$ for various $a$ for both polarized lights, which can be attributed to the reshaped LMRs due to the small size of the horizontal axis. Secondly, the $Q_{\text {abs }}$ spectra of the ENWs with large $a=400$ and $200 \mathrm{~nm}$ show some enhanced absorption sites owing to the superposition of resonant peaks, which may result from different LMR modes caused by the size difference between the horizontal and vertical axes. These enhanced absorption sites are greatly strengthened and tend to blue-shift with decreasing $a=400 \rightarrow 200 \mathrm{~nm}$, and then the resonant peaks for TM light exhibit similar peaks with the CNWs, while those for TE light present some new peaks utterly different from the CNWs with further deceasing $a=200 \rightarrow 100 \mathrm{~nm}$. For example, the number of resonant peaks decreases, and the peaks tend to blue-shift, but the light absorption is dramatically enhanced. Thirdly, with decreasing $a=400$ $\rightarrow 100 \mathrm{~nm}$, the light absorption is significantly enhanced in the short-wavelength range of $\lambda<\sim 420 \mathrm{~nm}$. Finally, The light absorption for TE light is much higher than that for TM light, resulting in a bigger photocurrent for TE light.

It is worth noting here that the light absorption near $\lambda=350 \mathrm{~nm}$ of the CNWs with small $D$ $(<100 \mathrm{~nm})$ is higher than that of the ENWs for TM light due to the excitation of the strongest $\mathrm{TM}_{01}$ and second strongest $\mathrm{TM}_{11} / \mathrm{TE}_{01}$ modes [29,38]; however, such small NWs are not practical for photovoltaic applications. In a word, these $Q_{\mathrm{abs}}$ spectra indicate that the light absorption of the ENWs with $b>a$ can be significantly enhanced compared to that of the CNWs.

\subsubsection{The ultimate photocurrent of Single ENWs}

To evaluate the light-trapping performance of the ENWs for photovoltaic applications, we now investigate the influence of geometrical parameters on the ultimate photocurrent $\left(J_{\mathrm{ph}}\right)$ calculated using Equation (8). In Figure 7a-c, we show $J_{\mathrm{ph}}$ as a function of $b(10 \rightarrow 1000 \mathrm{~nm})$ of the ENWs with $a=400$, 200 and $100 \mathrm{~nm}$ and the CNWs with $D=b$ for TM, TE and unpolarized light illumination, respectively. It is shown that for $b<a$, with increasing $b, J_{\mathrm{ph}}$ of the ENWs with small $b(<100 \mathrm{~nm})$ is much smaller than that of the CNWs due to the excitation of the strongest $\mathrm{TM}_{01}$ and second strongest $\mathrm{TM}_{11} / \mathrm{TE}_{01}$ modes of the CNWs $[29,38]$ and then can be slightly enhanced when $b \rightarrow a$ due to the superposition of resonant peaks, this enhancement is more evident for large than small $a$. More importantly, for $b$ $>a$ (except for $a \rightarrow b$ ), with increasing $b, J_{\mathrm{ph}}$ of the ENWs is dramatically enhanced compared to the CNWs. Specifically, $J_{\mathrm{ph}}$ periodically increases for large $a=400 \rightarrow 200 \mathrm{~nm}$ and linearly increases for small $a=100 \mathrm{~nm}$.

In the insets of Figure 7, we show the normalized G profiles of a CNW and three ENWs for TM and TE light illumination, respectively. Note here that $D=b=1000 \mathrm{~nm}$, and $a=400,200$ and $100 \mathrm{~nm}$, respectively. As presented above, the absorption enhancement of the ENWs only occurs near the vertical axis, leading to the small photocurrent enhancement with increasing $b$. However, with the symmetry breaking from CNWs to ENWs $(a=1000 \rightarrow 400 \rightarrow 200 \rightarrow 100 \mathrm{~nm}$ ), the LMR modes are reshaped due to the size decrease of the horizontal axis, and the absorption enhancement sites better fill in the whole ENWs for both TM and TE lights, especially TE light. 


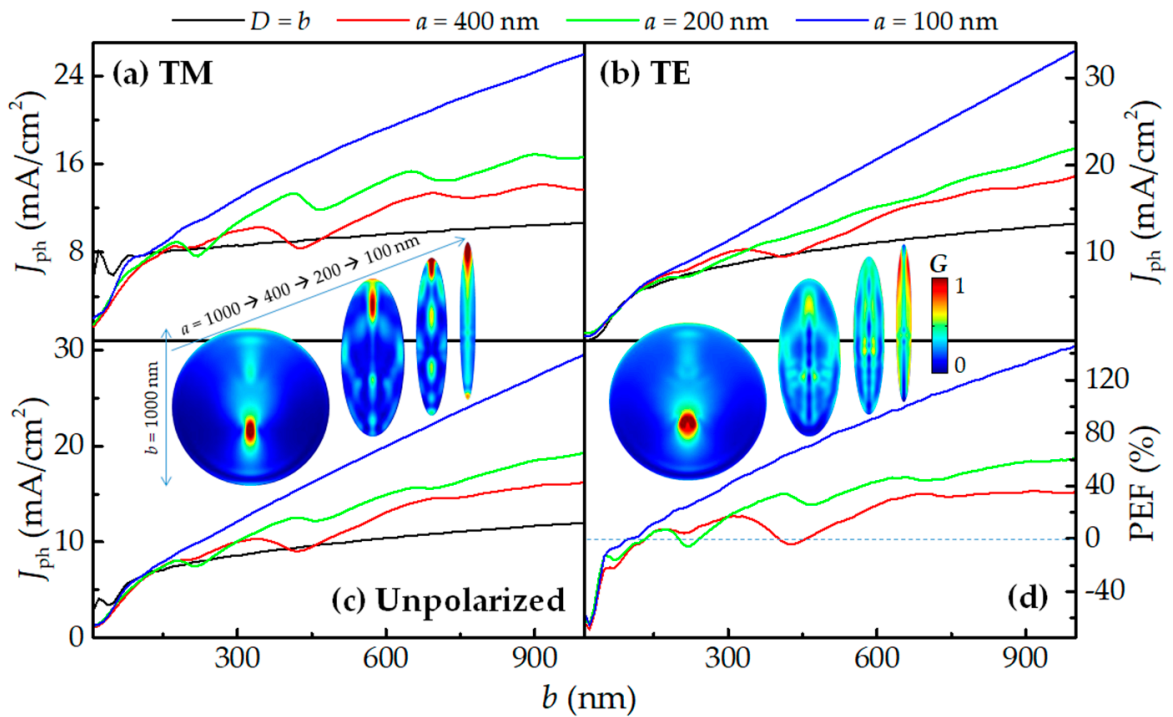

Figure 7. $J_{\mathrm{ph}}$ versus $b$ of the CNWs with $D=b$ and the ENWs with $a=400,200$ and $100 \mathrm{~nm}$ for (a) TM, (b) TE and (c) unpolarized light illumination, respectively. (d) The photocurrent enhancement factor (PEF) versus $b$ of the ENWs compared to the CNWs. The insets illustrate the normalized $G$ profiles of the CNWs with $D=b=1000 \mathrm{~nm}$ and the ENWs with $b=1000 \mathrm{~nm}$ and $a=400,200$ and $100 \mathrm{~nm}$ for TM and TE light illumination (with an identical color scale), respectively.

Finally, we show in Figure $5 \mathrm{c}$ the photocurrent enhancement factors (PEFs) defined by Equation (9). It is readily observed that $J_{\mathrm{ph}}$ of the ENWs with $b>a$ (except for $a \rightarrow b$ ) is much larger than the CNWs. In particular, $J_{\mathrm{ph}}$ reaches $29.53,19.32$ and $16.23 \mathrm{~mA} / \mathrm{cm}^{2}$ for $a=100,200$ and $400 \mathrm{~nm}$, which is $146.1 \%, 61.0 \%$ and $35.3 \%$ much larger than that of the CNW with $D=b=1800 \mathrm{~nm}\left(12.00 \mathrm{~mA} / \mathrm{cm}^{2}\right)$ due to the reshaped LMRs caused by the small size of the horizontal axis and $374.0 \%, 150.3 \%$ and $74.1 \%$ much larger than that of the CNW with $D=a=100\left(6.23 \mathrm{~mA} / \mathrm{cm}^{2}\right), 200\left(7.72 \mathrm{~mA} / \mathrm{cm}^{2}\right)$ and 400 $\left(9.32 \mathrm{~mA} / \mathrm{cm}^{2}\right)$ due to the increased light path length by the vertical axis, respectively.

\section{Conclusions}

In summary, we demonstrated the enhanced light trapping from the CNW to ENW. The influences of the geometrical parameters of the ENW on the light-trapping performance were numerically investigated. It was found that the elliptical cross-section can lead to significantly improved light trapping. The examination of the spatial profiles of the electric field, absorption mode and photogeneration rate revealed that the enhancement effect resulted from the symmetry-broken structure, which can simultaneously realize the increase of the light path length by the vertical axis and the reshaped LMRs by the horizontal axis. The simulation results showed that the photocurrent was significantly enhanced by $374.0 \%(150.3 \%, 74.1 \%)$ or $146.1 \%(61.0 \%, 35.3 \%)$ in comparison with that of the CNW with the same diameter as the horizontal axis of $100(200,400) \mathrm{nm}$ or the vertical axis of $1000 \mathrm{~nm}$, respectively. Therefore, such an elliptical nanowire can be applied to various semiconductors to improve light trapping and provides a promising approach for the future development of high-efficiency single NW solar cells.

Author Contributions: Conceptualization, W.L. and Y.Y.; methodology, W.L. and Y.W.; software, W.L. and X.W.; investigation, W.L. and X.G.; writing — original draft preparation, W.L and J.S.; writing—review and editing, W.L. and Y.Y. All authors have read and agreed to the published version of the manuscript.

Funding: This research was funded by the National Natural Science Foundation of China (Grant No. 61404057, 51801068 and 11875284), Key disciplines Fund for Optical Engineering in Henan Province (Grant No. 2018119) and Key Youth Teacher of Universities in Henan Province (Grant No. 2016GGJS-145).

Acknowledgments: We would like to thank the China Scholarship Council (Grant No. 201902720047) and the Master's Program on Energy and Power Engineering at Huanghuai University. 
Conflicts of Interest: The authors declare no conflict of interest.

\section{References}

1. Tian, B.; Zheng, X.; Kempa, T.J.; Fang, Y.; Yu, N.; Yu, G.; Huang, J.; Lieber, C.M. Coaxial silicon nanowires as solar cells and nanoelectronic power sources. Nature 2007, 449, 885-889. [CrossRef] [PubMed]

2. Nehra, M.; Dilbaghi, N.; Marrazza, G.; Kaushik, A.; Abolhassani, R.; Mishra, Y.K.; Kim, K.H.; Kumar, S. 1D semiconductor nanowires for energy conversion, harvesting and storage applications. Nano Energy 2020, 76, 104991. [CrossRef]

3. Li, Z.; Tan, H.H.; Jagadish, C.; Fu, L. III-V Semiconductor Single Nanowire Solar Cells: A Review. Adv. Mater. Technol. 2018, 3, 1800005. [CrossRef]

4. Kempa, T.J.; Day, R.W.; Kim, S.-K.; Park, H.-G.; Lieber, C.M. Semiconductor nanowires: A platform for exploring limits and concepts for nano-enabled solar cells. Energy Environ. Sci. 2013, 6, 719-733. [CrossRef]

5. Christesen, J.D.; Zhang, X.; Pinion, C.W.; Celano, T.A.; Flynn, C.J.; Cahoon, J.F. Design principles for photovoltaic devices based on Si nanowires with axial or radial p-n junctions. Nano Lett. 2012, 12, 6024-6029. [CrossRef]

6. Zhan, Y.; Li, X.; Li, Y. Numerical Simulation of Light-Trapping and Photoelectric Conversion in Single Nanowire Silicon Solar Cells. IEEE J. Sel. Top. Quantum Electron. 2013, 19, 1-8. [CrossRef]

7. Tian, B.; Kempa, T.J.; Lieber, C.M. Single nanowire photovoltaics. Chem. Soc. Rev. 2009, 38, 16-24. [CrossRef]

8. Tang, J.; Huo, Z.; Brittman, S.; Gao, H.; Yang, P. Solution-processed core-shell nanowires for efficient photovoltaic cells. Nat. Nanotechnol. 2011, 6, 568-572. [CrossRef]

9. Holm, J.V.; Jørgensen, H.I.; Krogstrup, P.; Nygård, J.; Liu, H.; Aagesen, M. Surface-passivated GaAsP single-nanowire solar cells exceeding 10\% efficiency grown on silicon. Nat. Commun. 2013, 4, 1498. [CrossRef]

10. Yuan, X.; Chen, X.; Yan, X.; Wei, W.; Zhang, Y.; Zhang, X. Absorption-Enhanced Ultra-Thin Solar Cells Based on Horizontally Aligned p-i-n Nanowire Arrays. Nanomaterials 2020, 10, 1111. [CrossRef]

11. Chen, W.; Roca i Cabarrocas, P. Rational design of nanowire solar cells: From single nanowire to nanowire arrays. Nanotechnology 2019, 30, 194002. [CrossRef] [PubMed]

12. Otnes, G.; Barrigón, E.; Sundvall, C.; Svensson, K.E.; Heurlin, M.; Siefer, G.; Samuelson, L.; Åberg, I.; Borgström, M.T. Understanding InP Nanowire Array Solar Cell Performance by Nanoprobe-Enabled Single Nanowire Measurements. Nano Lett. 2018, 18, 3038-3046. [CrossRef] [PubMed]

13. Song, K.-D.; Kempa, T.J.; Park, H.-G.; Kim, S.-K. Laterally assembled nanowires for ultrathin broadband solar absorbers. Opt. Express 2014, 22, A992-A1000. [CrossRef] [PubMed]

14. Zhang, X.; Pinion, C.W.; Christesen, J.D.; Flynn, C.J.; Celano, T.A.; Cahoon, J.F. Horizontal Silicon Nanowires With Radial p-n Junctions: A Platform for Unconventional Solar Cells. J. Phys. Chem. Lett. 2013, 4, 2002-2009. [CrossRef] [PubMed]

15. Floris, F.; Fornasari, L.; Bellani, V.; Marini, A.; Banfi, F.; Marabelli, F.; Beltram, F.; Ercolani, D.; Battiato, S.; Sorba, L.; et al. Strong Modulations of Optical Reflectance in Tapered Core-Shell Nanowires. Materials 2019, 12, 3572. [CrossRef]

16. Floris, F.; Fornasari, L.; Marini, A.; Bellani, V.; Banfi, F.; Roddaro, S.; Ercolani, D.; Rocci, M.; Beltram, F.; Cecchini, M. Self-Assembled InAs Nanowires as Optical Reflectors. Nanomaterials 2017, 7, 400. [CrossRef]

17. Zhu, L.; Hazama, Y.; Reddy, A.; Watanabe, K.; Nakano, Y.; Sugiyama, M.; Akiyama, H. Modeling and design for low-cost multijunction solar cell via light-trapping rear texture technique: Applied in InGaP/GaAs/InGaAs triple junction. Prog. Photovolt. Res. Appl. 2020, 28, 251-265. [CrossRef]

18. Peter Amalathas, A.; Alkaisi, M.M. Nanostructures for Light Trapping in Thin Film Solar Cells. Micromachines 2019, 10, 619. [CrossRef]

19. Hao, J.; Hao, H.; Li, J.; Shi, L.; Zhong, T.; Zhang, C.; Dong, J.; Xing, J.; Liu, H.; Zhang, Z. Light Trapping Effect in Perovskite Solar Cells by the Addition of Ag Nanoparticles, Using Textured Substrates. Nanomaterials 2018, 8, 815. [CrossRef]

20. Martinsen, F.A.; Smeltzer, B.K.; Ballato, J.; Hawkins, T.; Jones, M.; Gibson, U.J. Light trapping in horizontally aligned silicon microwire solar cells. Opt. Express 2015, 23, A1463-A1471. [CrossRef]

21. Kim, S.J.; Thomann, I.; Park, J.; Kang, J.H.; Vasudev, A.P.; Brongersma, M.L. Light Trapping for Solar Fuel Generation with Mie Resonances. Nano Lett. 2014, 14, 1446-1452. [CrossRef] [PubMed] 
22. Yu, K.J.; Gao, L.; Park, J.S.; Lee, Y.R.; Cocoran, C.J.; Nuzzo, R.G.; Chanda, D.; Rogers, J.A. Light Trapping in Ultrathin Monocrystalline Silicon Solar Cells. Adv. Energy Mater. 2013, 3, 1401-1406. [CrossRef]

23. Rothemund, R.; Umundum, T.; Meinhardt, G.; Hingerl, K.; Fromherz, T.; Jantsch, W. Light trapping in pyramidally textured crystalline silicon solar cells using back-side diffractive gratings. Prog. Photovolt. Res. Appl. 2013, 21, 747-753. [CrossRef]

24. Callahan, D.M.; Munday, J.N.; Atwater, H.A. Solar cell light trapping beyond the ray optic limit. Nano Lett. 2012, 12, 214-218. [CrossRef] [PubMed]

25. Liu, W.; Oh, J.I.; Shen, W.Z. Light Trapping in Single Coaxial Nanowires for Photovoltaic Applications. IEEE Electron. Device Lett. 2011, 32, 45-47. [CrossRef]

26. Ferry, V.E.; Polman, A.; Atwater, H.A. Modeling light trapping in nanostructured solar cells. ACS Nano 2011, 5, 10055-10064. [CrossRef] [PubMed]

27. Garnett, E.; Yang, P. Light Trapping in Silicon Nanowire Solar Cells. Nano Lett. 2010, 10, 1082-1087. [CrossRef]

28. Cao, L.; White, J.S.; Park, J.-S.; Schuller, J.A.; Clemens, B.M.; Brongersma, M.L. Engineering light absorption in semiconductor nanowire devices. Nat. Mater. 2009, 8, 643-647. [CrossRef]

29. Cao, L.; Fan, P.; Vasudev, A.P.; White, J.S.; Yu, Z.; Cai, W.; Schuller, J.A.; Fan, S.; Brongersma, M.L. Semiconductor Nanowire Optical Antenna Solar Absorbers. Nano Lett. 2010, 10, 439-445. [CrossRef]

30. Brönstrup, G.; Jahr, N.; Leiterer, C.; Csáki, A.; Fritzsche, W.; Christiansen, S. Optical properties of individual silicon nanowires for photonic devices. ACS Nano 2010, 4, 7113-7122. [CrossRef]

31. Kim, S.; Cahoon, J.F. Geometric Nanophotonics: Light Management in Single Nanowires through Morphology. Acc. Chem. Res. 2019, 52, 3511-3520. [CrossRef] [PubMed]

32. Choi, J.S.; Kim, K.-H.; No, Y.-S. Spatially localized wavelength-selective absorption in morphology-modulated semiconductor nanowires. Opt. Express 2017, 25, 22750-22759. [CrossRef]

33. Zhang, C.; Yang, Z.; Wu, K.; Li, X. Design of asymmetric nanovoid resonator for silicon-based single-nanowire solar absorbers. Nano Energy 2016, 27, 611-618. [CrossRef]

34. Luo, S.; Yu, W.B.; He, Y.; Ouyang, G. Size-dependent optical absorption modulation of Si/Ge and Ge/Si core/shell nanowires with different cross-sectional geometries. Nanotechnology 2015, 26, 085702. [CrossRef]

35. Kim, S.-K.; Song, K.-D.; Kempa, T.J.; Day, R.W.; Lieber, C.M.; Park, H.-G. Design of Nanowire Optical Cavities as Efficient Photon Absorbers. ACS Nano 2014, 8, 3707-3714. [CrossRef]

36. Kim, S.-K.; Day, R.W.; Cahoon, J.F.; Kempa, T.J.; Song, K.-D.; Park, H.-G.; Lieber, C.M. Tuning Light Absorption in Core/Shell Silicon Nanowire Photovoltaic Devices through Morphological Design. Nano Lett. 2012, 12, 4971-4976. [CrossRef] [PubMed]

37. Kempa, T.J.; Cahoon, J.F.; Kim, S.-K.; Day, R.W.; Bell, D.C.; Park, H.-G.; Lieber, C.M. Coaxial multishell nanowires with high-quality electronic interfaces and tunable optical cavities for ultrathin photovoltaics. Proc. Natl. Acad. Sci. USA 2012, 109, 1407-1412. [CrossRef] [PubMed]

38. Liu, W.; Oh, J.I.; Shen, W.Z. Light absorption mechanism in single c-Si (core)/a-Si (shell) coaxial nanowires. Nanotechnology 2011, 22, 125705. [CrossRef]

39. Liu, W.; Guo, X.; Xing, S.; Yao, H.; Wang, Y.; Bai, L.; Wang, Q.; Zhang, L.; Wu, D.; Zhang, Y.; et al. Off-Resonant Absorption Enhancement in Single Nanowires via Graded Dual-Shell Design. Nanomaterials 2020, 10, 1740. [CrossRef] [PubMed]

40. Zhong, Z.; Li, Z.; Gao, Q.; Li, Z.; Peng, K.; Li, L.; Mokkapati, S.; Vora, K.; Wu, J.; Zhang, G.; et al. Efficiency enhancement of axial junction InP single nanowire solar cells by dielectric coating. Nano Energy 2016, 28, 106-114. [CrossRef]

41. Solanki, A.; Gentile, P.; Boutami, S.; Calvo, V.; Pauc, N. Dielectric Coating-Induced Absorption Enhancement in Si Nanowire Junctions. Adv. Opt. Mater. 2015, 3, 120-128. [CrossRef]

42. Kim, S.-K.; Zhang, X.; Hill, D.J.; Song, K.-D.; Park, J.-S.; Park, H.-G.; Cahoon, J.F. Doubling Absorption in Nanowire Solar Cells with Dielectric Shell Optical Antennas. Nano Lett. 2015, 15, 753-758. [CrossRef] [PubMed]

43. Yu, Y.; Ferry, V.E.; Alivisatos, A.P.; Cao, L. Dielectric Core-Shell Optical Antennas for Strong Solar Absorption Enhancement. Nano Lett. 2012, 12, 3674-3681. [CrossRef] [PubMed]

44. Li, X.; Zhan, Y.; Wang, C. Broadband enhancement of coaxial heterogeneous gallium arsenide single-nanowire solar cells. Prog. Photovolt. Res. Appl. 2015, 23, 628-636. [CrossRef] 
45. Yang, Z.; Cao, G.; Shang, A.; Lei, D.Y.; Zhang, C.; Gao, P.; Ye, J.; Li, X. Enhanced Photoelectrical Response of Hydrogenated Amorphous Silicon Single-Nanowire Solar Cells by Front-Opening Crescent Design. Nanoscale Res. Lett. 2016, 11, 233. [CrossRef]

46. Yang, Z.; Li, X.; Lei, D.Y.; Shang, A.; Wu, S. Omnidirectional absorption enhancement of symmetry-broken crescent-deformed single-nanowire photovoltaic cells. Nano Energy 2015, 13, 9-17. [CrossRef]

47. Zhang, C.; Yang, Z.; Shang, A.; Wu, S.; Zhan, Y.; Li, X. Improved optical absorption of silicon single-nanowire solar cells by off-axial core/shell design. Nano Energy 2015, 17, 233-240. [CrossRef]

48. Shi, L.; Zhou, Z.; Huang, Z. The influence of silver core position on enhanced photon absorption of singlenanowire a-Si solar cells. Opt. Express 2013, 21, A1007-A1017. [CrossRef] [PubMed]

49. Zhou, J.; Zhang, Z.; Wu, Y.; Xia, Z.; Qin, X. Significantly enhanced coupling to half-space irradiation using a partially capped nanowire for solar cells. Nano Energy 2018, 45, 61-67. [CrossRef]

50. Leahu, G.; Petronijevic, E.; Belardini, A.; Centini, M.; Sibilia, C.; Hakkarainen, T.; Koivusalo, E.; Rizzo Piton, M.; Suomalainen, S.; Guina, M. Evidence of Optical Circular Dichroism in GaAs-Based Nanowires Partially Covered with Gold. Adv. Opt. Mater. 2017, 5, 1601063. [CrossRef]

51. Mann, S.A.; Garnett, E.C. Extreme Light Absorption in Thin Semiconductor Films Wrapped around Metal Nanowires. Nano Lett. 2013, 13, 3173-3178. [CrossRef] [PubMed]

52. Jia, Y.; Qiu, M.; Wu, H.; Cui, Y.; Fan, S.; Ruan, Z. Theory of Half-space Light Absorption Enhancement for Leaky Mode Resonant Nanowires. Nano Lett. 2015, 15, 5513-5518. [CrossRef] [PubMed]

53. Wang, B.; Leu, P.W. Enhanced absorption in silicon nanocone arrays for photovoltaics. Nanotechnology 2012, 23, 194003. [CrossRef] [PubMed]

54. Wang, B.; Stevens, E.; Leu, P.W. Strong broadband absorption in GaAs nanocone and nanowire arrays for solar cells. Opt. Express 2014, 22, A386-A395. [CrossRef] [PubMed]

55. Anttu, N.; Mäntynen, H.; Sorokina, A.; Kivisaari, P.; Sadi, T.; Lipsanen, H. Geometry Tailoring of Emission from Semiconductor Nanowires and Nanocones. Photonics 2020, 7, 23. [CrossRef]

56. Zhou, L.; Yu, X.; Zhu, J. Metal-Core/Semiconductor-Shell Nanocones for Broadband Solar Absorption Enhancement. Nano Lett. 2014, 14, 1093-1098. [CrossRef] [PubMed]

57. Xu, Z.; Huangfu, H.; He, L.; Wang, J.; Yang, D.; Guo, J.; Wang, H. Light-trapping properties of the Si inclined nanowire arrays. Opt. Commun. 2017, 382, 332-336. [CrossRef]

58. Kayes, M.; Leu, P. Comparative study of absorption in tilted silicon nanowire arrays for photovoltaics. Nanoscale Res. Lett. 2014, 9, 620. [CrossRef]

59. Hong, L.; Rusli; Wang, X.; Zheng, H.; Wang, H.; Yu, H. Design guidelines for slanting silicon nanowire arrays for solar cell application. J. Appl. Phys. 2013, 114, 084303-084306. [CrossRef]

60. Adibzadeh, F.; Olyaee, S. Optical absorption enhancement in vertical InP nanowire random structures for photovoltaic applications. Opt. Quantum Electron. 2020, 52, 6. [CrossRef]

61. Qin, X.; Wu, Y.; Zhang, Z.; Xia, Z.; Zhou, J.; Zhu, J. Broadband light absorption enhancement in randomly rotated elliptical nanohole arrays for photovoltaic application. Appl. Opt. 2019, 58, 1152-1157. [CrossRef] [PubMed]

62. Zhang, H.; Kramarenko, M.; Osmond, J.; Toudert, J.; Martorell, J. Natural Random Nanotexturing of the Au Interface for Light Backscattering Enhanced Performance in Perovskite Solar Cells. ACS Photonics 2018, 5, 2243-2250. [CrossRef]

63. Dhindsa, N.; Saini, S.S. Comparison of ordered and disordered silicon nanowire arrays: Experimental evidence of photonic crystal modes. Opt. Lett. 2016, 41, 2045-2048. [CrossRef] [PubMed]

64. Bao, H.; Ruan, X. Optical absorption enhancement in disordered vertical silicon nanowire arrays for photovoltaic applications. Opt. Lett. 2010, 35, 3378-3380. [CrossRef]

65. Sychugov, I.; Sangghaleh, F.; Bruhn, B.; Pevere, F.; Luo, J.W.; Zunger, A.; Linnros, J. Strong Absorption Enhancement in Si Nanorods. Nano Lett. 2016, 16, 7937-7941. [CrossRef]

66. Kolezas, G.D.; Zouros, G.P.; Pagiatakis, G.K.; Roumeliotis, J.A. All-Anisotropic Spheroidal Photonic Antennas: Theory and Modeling. IEEE J. Sel. Top. Quantum Electron. 2020, 27, 8300312. [CrossRef]

67. Wu, Y.; Xia, Z.; Liang, Z.; Zhou, J.; Jiao, H.; Cao, H.; Qin, X. Broadband absorption enhancement in elliptical silicon nanowire arrays for photovoltaic applications. Opt. Express 2014, 22, A1292-A1302. [CrossRef]

68. Qin, X.; Xia, Z.; Wu, Y.; Zhou, J.; Zhang, Z. Enhanced light absorption in perpendicular elliptical silicon nanocone array for solar cells. Appl. Opt. 2017, 56, 2307-2313. [CrossRef] 
69. Xia, Z.; Qin, X.; Wu, Y.; Pan, Y.; Zhou, J.; Zhang, Z. Efficient broadband light absorption in elliptical nanohole arrays for photovoltaic application. Opt. Lett. 2015, 40, 5814-5817. [CrossRef]

70. Palik, E.D. Handbook of Optical Constants of Solids; Academic Press: London, UK, 1985.

71. Kane, Y. Numerical Solution of Initial Boundary Value Problems Involving Maxwell's Equations in Isotropic Media. IEEE Trans. Antennas Propag. 1966, 14, 302-307. [CrossRef]

72. Taflove, A.; Hagness, S.C. Computational Electrodynamics: The Finite-Difference Time-Domain Method, 3rd ed.; Artech House: Norwood, MA, USA, 2005.

73. Ee, H.S.; Song, K.D.; Kim, S.K.; Park, H.G. Finite-Difference Time-Domain Algorithm for Quantifying Light Absorption in Silicon Nanowires. Isr. J. Chem. 2012, 52, 1027-1036. [CrossRef]

74. Bohren, C.F.; Huffman, D.R. Absorption and Scattering of Light by Small Particles; John Wiley \& Sons, Inc.: New York, NY, USA, 1998.

75. Munday, J.N.; Atwater, H.A. Large integrated absorption enhancement in plasmonic solar cells by combining metallic gratings and antireflection coatings. Nano Lett. 2010, 11, 2195-2201. [CrossRef] [PubMed]

Publisher's Note: MDPI stays neutral with regard to jurisdictional claims in published maps and institutional affiliations.

(C) 2020 by the authors. Licensee MDPI, Basel, Switzerland. This article is an open access article distributed under the terms and conditions of the Creative Commons Attribution (CC BY) license (http://creativecommons.org/licenses/by/4.0/). 